\title{
The Relationship of Rapid Eye Movement Sleep Behavior Disorder and Freezing of Gait in Parkinson's Disease
}

\author{
Chelsea Mae N. Nobleza ${ }^{1}$, Mariah Siddiqui ${ }^{2,1}$, Parth V. Shah ${ }^{3}$, Prachi Balani ${ }^{4}$, Angel R. Lopez ${ }^{5}$, Safeera \\ Khan $^{4}$ \\ 1. Neurology, California Institute of Behavioral Neurosciences \& Psychology, Fairfield, USA 2. Neurology, St. George's \\ University, True Blue, GRD 3. Medicine, California Institute of Behavioral Neurosciences \& Psychology, Fairfield, USA \\ 4. Internal Medicine, California Institute of Behavioral Neurosciences \& Psychology, Fairfield, USA 5. Psychiatry, \\ California Institute of Behavioral Neurosciences \& Psychology, Fairfield, USA
}

Corresponding author: Chelsea Mae N. Nobleza, kelooogs@gmail.com

\begin{abstract}
Rapid eye movement sleep behavior disorder (RBD) contributes to injury due to the alteration of the expected atonia during rapid eye movement (REM) sleep. It occurs before the overt signs of Parkinson's disease (PD). The co-expression of PD and RBD is characterized by non-tremor predominant subtype and higher incidence of freezing. Freezing of gait (FOG) is a debilitating symptom seen in PD patients that lead to falls. While this phenomenon is understood poorly, the involvement of the pedunculopontine nucleus (PPN) and the neural circuits that control locomotion and gait have been examined. This network has also the same control for REM sleep and arousal. The close relationship between PD and RBD and FOG's consequences has led us to explore the relationship between RBD and PD with FOG. This review provides an overview of the neural connections that control gait, locomotion, and REM sleep. The neural changes were seen in PD with FOG and RBD, and sensory and motor changes observed in these two diseases. The functional neuroanatomy that controls REM sleep, arousal, and locomotion overlap significantly with multiple neural circuits affected in RBD and PD with FOG. Visual perception dysfunction and motor symptoms that primarily affect gait initiation are common to both patients with RBD and FOG in PD, leading to freezing episodes. Prospective studies should be conducted to elucidate the relationship of RBD and PD with FOG subtype and find innovative treatment approaches and diagnostic tools for PD with FOG.
\end{abstract}

Categories: Neurology

Keywords: freezing of gait, parkinson's disease, gait, postural instability, pedunculopontine nucleus, visuoperceptive abnormalities, anticipatory postural adjustment, rbd

\section{Introduction And Background}

Received 10/26/2020 Review began $11 / 10 / 2020$ Review ended 12/24/2020 Published 12/30/2020

\section{(c) Copyright 2020} Nobleza et al. This is an open access article distributed under the terms of the Creative Commons Attribution License CC-BY 4.0., which permits unrestricted use, distribution, and reproduction in any medium, provided the original author and source are credited.
Rapid eye movement behavior sleep disorder (RBD) is characterized by a loss of normal muscle atonia and subsequent dream enactment [1]. It occurs with other synucleinopathies like Parkinson's disease (PD), multisystem atrophy, and Lewy body dementia in up to $81 \%$ of patients from five to 29 years [2]. RBD likely happens before the overt motor, cognitive and autonomic impairments in PD proposed by Braak's staging system, which postulates that medullary structures are affected first in synucleinopathies and eventually ascend to more rostral structures; specifically, a prominent degeneration in the sublaterodorsal nucleus (SLD) will cause rapid eye movement (REM) sleep without atonia and RBD [3, 4]. Polysomnographic studies have shown that $58 \%$ of patients with PD have some loss of muscle atonia during REM sleep [5]. This finding is congruent to the study conducted by Sixel-Döring F et al., where they found that $46 \%$ of all PD patients had RBD, as diagnosed by polysomnography with video synchronization [6].

Patients with both PD and RBD exhibit a non-tremor-predominant subtype of the disease, and a higher incidence of freezing of gait (FOG), leading to increased frequency of falls [7, 8]. FOG is a debilitating symptom with a complex mechanism understood poorly, occurring in one-third of patients with Parkinson's disease. FOG is defined by an episodic inability for the feet to move forward, despite the intent to walk with predetermined stride length. FOG can have motor phenomena associated with initiation disturbances and transient interruption to stepping while walking. Theories that explain FOG include ineffective gait patterns, alteration in anticipatory postural adjustments (APA), leading to problems in step initiation, perceptual and frontal lobe area dysfunction, disturbed central drive disruptions, and automaticity of gait [9]. While the etiology of FOG is complex, existing evidence states that FOG involves a disruption in neuroanatomical networks in the brainstem, particularly the pedunculopontine nucleus (PPN), a component of the mesencephalic locomotor center postural control circuits responsible for gait and locomotion as well as reward, motivation, compulsion, and REM sleep [10, 11]. Recent work has shown that PPN grey matter atrophy is more evident in patients with idiopathic PD with FOG than those without FOG [12]. These anatomical networks are also involved in RBD's pathophysiology through its thalamic connections to the medial prefrontal and anterior cingulate cortices $[10,13,14]$. It is hypothesized that deficits arising from dysfunction in these regions, such as sleep disorders, are expected to co-express in patients with PD and 


\section{Cureus}

FOG [8]. The relationship between RBD and Parkinson's disease has been widely explored. Still, only a few published studies elucidate the relationship between RBD and FOG in PD, considering how freezing negatively impacts these patients. This review will provide an overview of the relationship between RBD and FOG by paying attention to the involvement of the PPN and other neural systems responsible for the loss of tone during REM sleep, locomotion, and posture, describe the changes that happen in these neural networks as a result of RBD and PD with FOG and, explore the gait, posture, and sensory and motor abnormalities shared by these two disease entities.

\section{Review}

\section{Gait, locomotion, and REM sleep control}

Gait control, locomotion, and REM sleep share common pathways in which the PPN is central to all these functional neuroanatomic connectivities. Figure 1 illustrates the overlap in these structures.

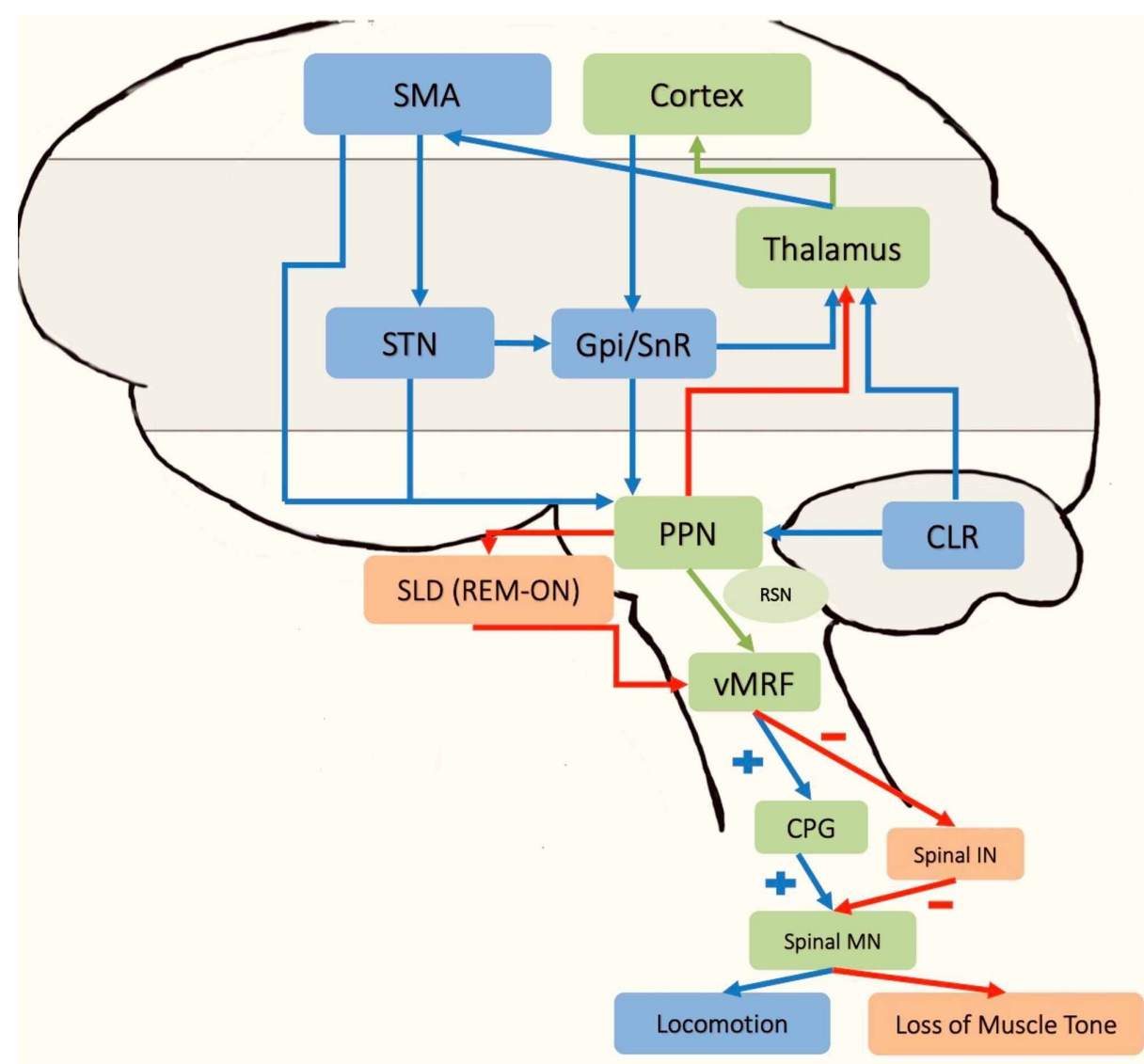

\section{FIGURE 1: Simplified diagram of the neuroanatomic connections that control arousal, REM sleep, and gait}

Blue-colored arrows and boxes represent connections exclusive to the motor locomotor region (posture and gait). Red-colored arrows and orange boxes represent the neural network exclusive for REM sleep control. In contrast, green-colored arrows and boxes represent the overlap between the MLR, arousal, and REM sleep control.

SMA - supplementary motor cortex; STN - subthalamic nucleus; Gpi/Snr - globus pallidus internus/substantia nigra pars reticulata; PPN - pedunculopontine nucleus; RSN - reticulospinal nucleus; SLD - sublaterodorsal nucleus; vMRF - ventromedial reticular formation; CPG - central pattern generators; CLR cerebellar locomotor region; Spinal MN - spinal motor neuron, Spinal IN - spinal interneurons; MLR - mesencephalic locomotor region; REM - rapid eye movement

The PPN in the mesopontine tegmentum is involved in locomotor modulation, postural muscle tone, arousal, and REM sleep atonia [15]. Cholinergic cells in the PPN project to the thalamus and increased acetylcholine transmission induces arousal [16]. The same cells have the activity in the REM on and offcenter in the brainstem [17]. High-frequency stimulation (>100 Hz) of the PPN results in a suppression of postural muscle tone instead of locomotion $[18,19]$. However, a slow stimulation brings the membrane potential firing from a rhythmic state bursting to a state of desynchronized firing observed during arousal or REM sleep [17]. The SLD mediates the loss of muscle tone during REM sleep through its cholinergic 
connections to the PPN [20]. The PPN is also a part of the mesencephalic locomotor region (MLR) involved in APA and subsequent gait initiation [21]. Acetylcholine stimulation of the caudal PPN (corresponds to the MLR) is associated with suppressing muscle tone via projections to the ventromedial medullary reticular formation (vmRF) while blocking the vmRF with atropine inhibited atonia and facilitated MLR-induced locomotion [22]. With the extensive involvement of the PPN in both REM sleep and locomotion, dysfunction in this area and other neural networks connected to this nucleus can alter REM sleep and poor execution of purposeful movement. The locomotor pathway also includes the reticulospinal nucleus (RSN), which regulates muscle tone. Modulation of RSN during the gait cycle involves both muscle-tone related RSNs and locomotor rhythm. Executing muscle tone regulation during movement requires the integration of all spinal reflex networks. These spinal reflex networks produce rhythm and locomotor movements by activating the central pattern generators (CPG). The CPGs also play a role during the stance phase of the gait cycle. Lastly, the integration of descending supraspinal signals and sensory afferents is necessary for effective control of posture [23]. While CPGs play a role in the gait cycle at the spinal level, purposeful gait requires executive function, attention, and judgment of one's immediate surroundings in which the supraspinal locomotor region (SLR) is involved [24]. This region primarily includes primary and supplementary motor cortices, prefrontal cortices, subcortical structures (basal ganglia, vmRF, MLR, and cerebellar locomotor region) [25]. The subthalamic nucleus (STN), in particular, has an essential role in locomotion control through its direct connection to the supplementary motor area (SMA) and downward projections to the MLR. The STN then serves as a gate to integrate cortical output and cerebellar information by activating or inhibiting the MLR through glutamatergic projections or basal ganglia gamma-aminobutyric acid (GABA)ergic output [26]. The MLR, in turn, relays the information to the central pattern generators (CPGs) [23, 26].

\section{Changes in the neural networks due to freezing of gait in PD and RBD}

Neural network changes as a result of FOG in PD and RBD have also been explored. A study by Pozzi et al. determined that the disruption in the communication in the cortical-subthalamic network in the hemisphere with decreased dopaminergic innervation led to gait freezing. This was observed during the transition from a well-executed gait cycle to the onset of gait freezing. Decoupling between the SMA and STN in the hemisphere with decreased dopaminergic innervation suggests asymmetry between the two hemispheres [27]. Asymmetry was also noted in the PPN and its connections to other locomotor regions in a study made by Fling et al., more notably in the right hemisphere frontal cortex and the PPN. Further, lateralization of the PPN tract volume towards the left hemisphere suggests a less accurate and longer time to initiate a task or inhibit an inappropriate action in patients with FOG [28]. Considering these neural networks' asymmetric involvement, will subsequent motor manifestations in these patients also have asymmetric presentations?

Two studies also found a significant reduction in the cerebellar locomotor region (CLR) connection to the PPN in FOG patients $[28,29]$. Fasano et al. state that $90 \%$ of the lesions in their study were functionally connected to the bilateral dorsal medial cerebellum in FOG patients and a separate anatomic structure that lead to asterixis or hemichorea [29]. These findings show a heterogeneous involvement of the neural networks that control REM sleep, locomotion, and posture.

Supraspinal control of gait is also affected in RBD and PD with FOG. One study revealed a decrease in PPN functional connectivity with the bilateral SMA proper, pre-SMA, and dentate nucleus in PD with impaired postural instability and RBD group. In particular, the PPN-SMA connectivity defect was seen in PD patients with a positive pull test and prolonged APA duration before gait initiation. PD with impaired postural instability and RBD patients also have decreased PPN functional connectivity with the bilateral medial prefrontal cortex (MPFC). This impaired connectivity is also evident in a study made by Wang et al., where patients with FOG have altered functional connectivity between the PPN and corticopontine-cerebellar pathways and visual temporal areas, including the tracts projecting to motor, sensory and cognitive regions. During tasks requiring high cognitive load, PD patients with FOG had weak activity in the pre-SMA [30]. In contrast, the PD patients without FOG could recruit the prefrontal areas, including the pre-SMA and the medial prefrontal cortex [31]. Simultaneous use of cognition while doing a motor task may lead to ineffective walking and subsequent freezing.

Patients with RBD were found to have altered functional connectivity between the bilateral PPN and the ventral posterior-anterior cingulate cortex (ACC), regions involved in arousal and alertness. The same structures, known as the functional associative network (ACC and SMA), serve as a compensation network in PD patients with faulty automatic control of sensorimotor networks [30]. However, with RBD and PD coexpression in several patients, this compensatory functional associative network may be rendered useless. Compensatory networks in patients with FOG are also observed in a study by Fling et al. The SMA involved in supraspinal control of gait initiation has increased connection with the MLR and CLR in patients with FOG. In contrast, FOG negative patients had significantly greater functional connectivity than FOG positive patients in the hyper-direct pathway between the STN and SMA. This increased connection implies a reorganization of functional communication within the locomotor network in FOG positive patients. Nevertheless, it fails to serve as a compensatory for freezing, as evidenced by the positive association between FOG severity ratings and increased functional connectivity in the MLR - SMA and CLR - SMA neural networks [32].

The observed neural changes in these networks do not localize to a single neuroanatomic structure but 


\section{Cureus}

rather reflect derangements of both spinal and supraspinal networks and, along with it, faulty compensatory mechanisms that worsen freezing. Figure 2 illustrates the changes observed.

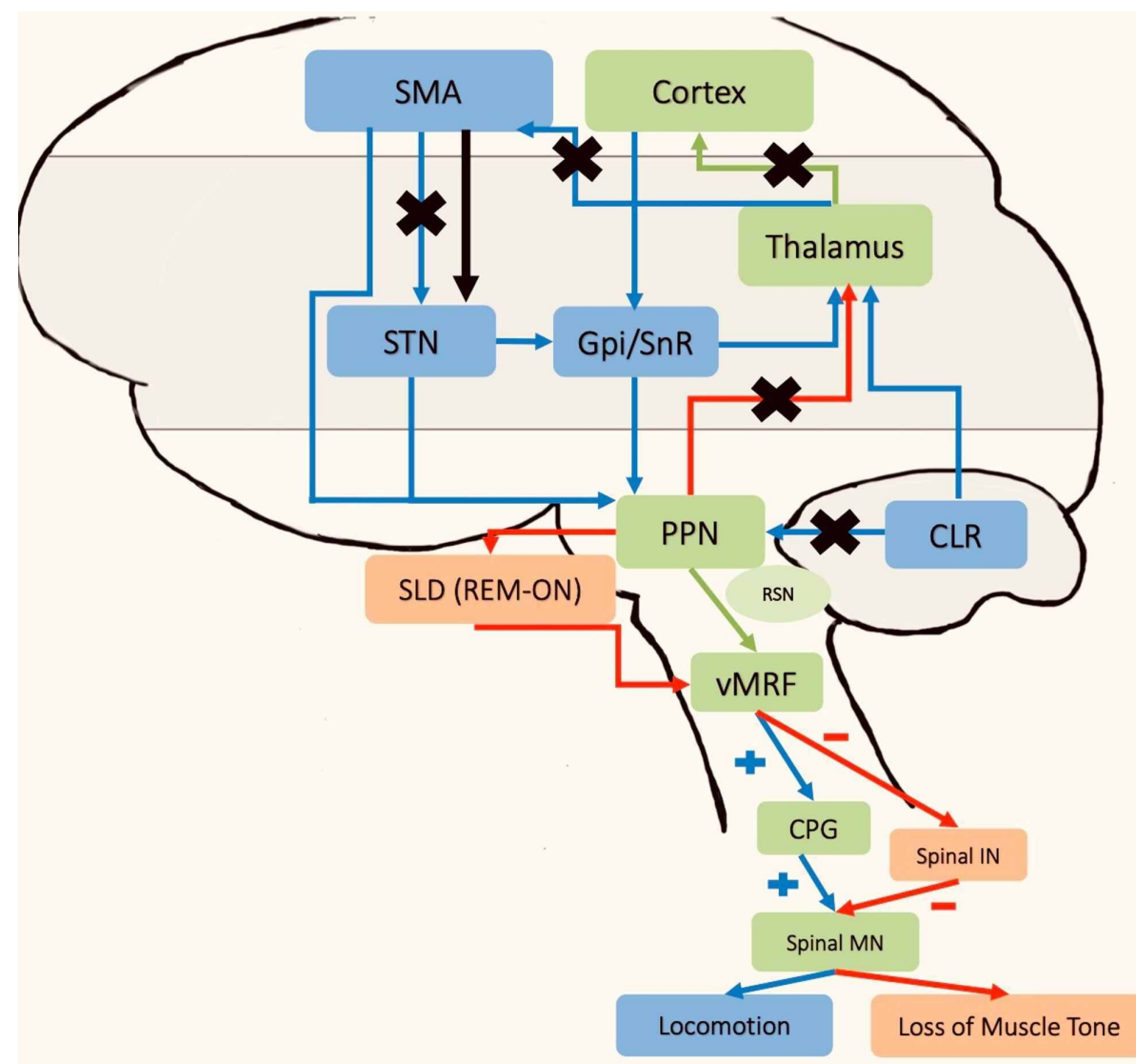

FIGURE 2: Changes in the neural networks seen in RBD and PD with FOG

The cross marks represent the altered connections as a result of RBD and PD in FOG. The black arrow represents the increased connectivity of the SMA to the MLR region, as seen in FOG patients.

SMA - supplementary motor cortex; STN - subthalamic nucleus, Gpi/Snr - globus pallidus internus/substancia nigra pars reticulata; PPN - pedunculopontine nucleus; RSN - reticulospinal nucleus; SLD - sublaterodorsal nucleus; vMRF - ventromedial reticular formation; CPG - central pattern generators; CLR - cerebellar locomotor region; Spinal MN - spinal motor neuron; Spinal IN - spinal interneurons; MLR - mesencephalic locomotor region; RBD - rapid eye movement sleep behavior disorder; PD - Parkinson's disease; FOG - freezing of gait

\section{Sensory and motor changes in RBD and FOG}

Sensory Changes

The integration of multiple environmental sensory inputs is necessary to execute a purposeful movement. Several studies have looked into these sensory interruptions that contribute to motor impairments in PD and RBD patients. FOG patients unresponsive to levodopa treatment have decreased visuospatial functioning performance as assessed using the Judgment of Line Orientation Test [33]. This visual perception impairment was also tested in FOG and FOG negative PD patients and controls using three sets of doorways with decreasing sizes. Gait velocity was slower in FOG patients during their initial encounter with the doorway. A significant decrease in step length and increased step length variability in FOG patients as they approached the narrow doorway, as well as an increased base of support, were noted [34]. These variables occurring before arrival at the doorway implies that impaired perceptual processes are interrupting the initially planned movement to pass the doorway, suggesting that visuospatial perception, which corresponds to the restructuring of the picture's inherent properties to obtain an overall, meaningful representation, enabling object identification and location determination, contributes to the pathophysiology of gait freezing $[34,35]$.

Interestingly, idiopathic RBD (iRBD) patients also experience visuoperceptual dysfunction based on poor performance in the Biederman fragmented picture identification task. Results from this test reveal a lack of 
visual priming, which, when present, generally leads to a faster response to a stimulus as a result of prior exposure. This response seen in iRBD patients is consistent with impaired intermediate perceptive processing of visual information. The initial presentation of an image triggers identifying the overall structural representation of the image [36]. Marques et al. support this finding. In their study, impairment in object identification in RBD using the identification thresholds of incomplete contour drawings of objects is noted, regardless of whether RBD is associated with PD [35]. With the occurrence of visuoperceptive dysfunction in either PD or RBD, it shows that the influence of visuoperceptive dysfunction during purposeful movement is heightened in patients with PD and co-morbid RBD.

One study also reports auditory and audiovisual perception changes in PD patients. Specifically, there is a significant difference between auditory and visual reaction times in $\mathrm{PD}$, with a more significant alteration observed in the FOG subtype. The responses to visual stimuli were significantly slower compared with the auditory modality [37]. This finding could be explained by the already altered visual perception in these patients, as observed in previous studies. However, it is notable that this difference increases with disease duration and the development of FOG, suggesting a possible compensatory role of the faster auditory reaction time to navigate one's immediate surroundings. Although multisensory facilitation occurs in PD, it is significantly less enhanced than age-matched healthy controls [37].

\section{Motor Changes}

Motor manifestations in patients with RBD and PD have been described. A study by Postuma et al. has shown that PD with RBD are non-tremor predominant [38], had increased frequency of falls, and had less response to levodopa [31]. Over time, other studies further characterized these findings by examining gait parameters and polysomnographic findings to establish the presence of rigidity and subsequent freezing in patients with PD and RBD.

Gait parameters were analyzed in various studies. In one study, patients with probable RBD exhibited fewer steps, increased swing time, and step length variability with a tendency to decrease their velocity and cadence [39]. Postural sway as an essential component of the gait cycle was also described by Chen et al., where idiopathic RBD patients showed unrefined sway, which was more evident during difficult situations, as evidenced by increased jerkiness. The noted decrease in sway's smoothness mirrors the nervous system's attempt to correct sway during upright posture. Patients with RBD exhibit decreased smoothness of sway, probably due to rigidity. They also found an increased variability during a forward trunk acceleration, which confers with other study findings [40]. The posterior shift of the center of pressure (CoP) is reduced during the gait cycle's anticipatory and propulsive phase in patients with RBD without coexisting PD [41]. The posterior displacement in CoP is an essential part of anticipatory postural adjustments (APA) to accelerate the body forward and move to the single-stance leg for stabilization before initiation of step [42]. Therefore, failure to shift the CoP leads to gait initiation failure, a higher probability of falls, and freezing. PD with RBD patients and those with RBD alone had longer APA duration [31]. Thevathasan et al. also revealed that patients with PD and FOG had freezing episodes during turning. These patients also needed a more extended time during the turning task. Also, cadence and step length decreased, and variability in step length increased [43]. The similar gait findings seen in both PD with FOG and probable RBD patients support the probability that shared pathophysiology exists between these two disease entities.

Increased muscle activity during REM sleep was found in PD with FOG and RBD patients. The tonic EMG activity, which generally should be low, considering that REM sleep is characterized by loss of muscle tone, increases PD with FOG than in PD without FOG patients [14]. This electromyography (EMG) finding means that REM sleep without atonia (RWSA) occurs more in these patients. RWSA correlates with the disease duration and severity in PD patients as well as RBD based on the RBD screening questionnaire [44]. The increased muscle tone during REM sleep leads to a decreased capacity to generate a posterior shift in the CoP [41]. Also, there is an increased symmetric forearm rigidity in PD with RWSA patients [45]. These findings reflect that RBD's presence in PD patients predisposes them to the FOG subtype, has more gait deficits, and increased rigidity. These findings could also mean that more extensive and earlier neural degeneration in the neural networks that control both REM sleep and locomotion in patients with PD FOG and coexisting RBD leads to a more severe disease presentation. Table 1 presents all the articles reviewed in this study.

\begin{tabular}{|c|c|c|c|c|}
\hline $\begin{array}{l}\text { Author and } \\
\text { year of } \\
\text { publication }\end{array}$ & $\begin{array}{l}\text { Number } \\
\text { of } \\
\text { patients }\end{array}$ & $\begin{array}{l}\text { Type } \\
\text { of } \\
\text { study }\end{array}$ & Results & Conclusion \\
\hline $\begin{array}{l}\text { Pozzi et al. } \\
2019 \text { [27] }\end{array}$ & $\begin{array}{l}\text { Seven } \\
\text { PD }\end{array}$ & $\begin{array}{l}\text { Cross- } \\
\text { section }\end{array}$ & $\begin{array}{l}\text { A notable decrease in communication between the } \\
\text { STN and cortex is evident before transitioning to } \\
\text { freezing and recovering from normal walking. }\end{array}$ & $\begin{array}{l}\text { FOG is a result of dysfunction in STN- } \\
\text { cortical communication. This finding } \\
\text { suggests faulty cortical-subcortical } \\
\text { circuitry, which could be a potential area } \\
\text { for neuromodulation to improve freezing. }\end{array}$ \\
\hline Fasano et al. & 14 PD & RCT & $\begin{array}{l}90 \% \text { of lesions in FOG patients have been located } \\
\text { in the CLR, specifically the dorsal medial }\end{array}$ & $\begin{array}{l}\text { This may guide the development of new } \\
\text { therapies for treatment-resistant FOG by }\end{array}$ \\
\hline
\end{tabular}




\section{Cureus}

2018 [29]

Gallea et al. 52 PD; 2017 [31] 25 controls

Crosssection

$\begin{array}{lll} & & \\ \text { Wang et al. } & 31 \mathrm{PD} & \\ 2016[30] & 16 & \text { Cross- } \\ & \text { controls } & \text { section }\end{array}$

26 PD

Fling et al patients; 2014 [28] 15 agematched controls

26 PD;

Fling et al. patients

2013 [32] matched

controls

15 PD;

Thevathasan nine

et al. 2012 age-

matched

controls

39 PD;

Fearon et al. 17 age- Cross-

2015 [37] matched section controls

Factor et al. 2014 [33] 135 PD

15 iRBD

Plomhause $30 \mathrm{PD}$

et al. 201420 age-

[36] matched

controls

20 PD;

10 iRBD;

Marques et eight Cross-

al. 2009 [35] age- section

matched

controls

31 PD;

Almeida et 16 age- Cross-

al. 2009 [34] matched section controls

61,41

Linn-Evans PD, 20

et al. 2020 age-

[45] cerebellum.

As noted in those with sleep disorder and impaired postural control, patients with longer APA have decreased connectivity in the PPN and the SMA and the PPN and ACC, the locomotor, and arousal regions.

FOG patients have decreased PPN and frontal cortex communication compared to FOG negative patients and controls. In particular, the corticopontine-cerebellar pathways are notably affected in addition to the visual temporal areas.

FOG+ patients have increased connectivity Cross- between the SMA and MLR and CLR than FOGsection patients and controls. This results in increased freezing severity.

There is decreased connectivity of the PPN with the cerebellum, thalamus, and multiple frontal cortex regions, particularly in the right hemisphere. More

section left hemisphere-lateralized PNN volume was noticed to have poorer performance on cognitive tasks in FOG patients.

Bilateral PPN stimulation improved gait freezing more than unilateral stimulation but not step length variability.

The PD group had significantly visuoperception ability than healthy controls. Still, auditory reaction times were significantly faster than visual for the PD group only. Multisensory facilitation occurs in PD but is less enhanced than in healthy controls.

The unresponsive FOG group had significantly Cross lower visuospatial ability and executive functioning Section than other groups. The responsive FOG group was found to exhibit hallucinations.

Idiopathic RBD patients lack visual priming effects as assessed by the Biederman task. Parkinson's disease patients with RBD had poorer visuoperceptive performance levels than PD patients without RBD.

There is poorer performance on object identification seen in PD patients with RBD and idiopathic RBD patients.

FOG group had step length variability as well as within-trial variability of step length and step time as a response to the narrow doorway.

Cross- Forearm rigidity was significantly higher and more section symmetric in the PD-RSWA+ group. identifying areas for DBS.

The pedunculopontine nucleus and neural networks involved in REM sleep and locomotion support the clinical manifestation and the relationship between RBD and postural control impairments in Parkinson's disease.

FOG in PD is associated with abnormal PPN and frontal cortex networks, mainly affecting the corticopontine-cerebellar pathways.

There is functional reorganization in the connections between SMA and MLR and the CLR, which leads to a faulty neural compensatory response during FOG.

FOG is strongly related to neuroanatomic deficits in the right hemisphere's locomotor network.

Bilateral stimulation of a caudal PPN region improves gait freezing but not step length variability.

There are significant sensory abnormalities in PD. Multisensory abnormalities are not related to disease duration and could be a potential biomarker for the disease.

The unresponsive FOG had executive and visuospatial dysfunction, while responsive FOG is associated with hallucinations suggesting posterior cortical regions' involvement.

There is intermediate visuoperceptive processing dysfunction in idiopathic RBD patients. RBD in PD is associated with decreased visuoperceptive abilities but not attention.

This perceptual dysfunction seen in RBD may not be related to the loss of dopamine innervation.

Freezing may have a perceptual pathology that influences neural movement planning.

In patients with mild to moderate PD and RSWA, there is associated increased and more symmetric presentation of upper limb rigidity. 


\section{Cureus}
Alibiglou et
al. 2016 [41]
30 PD
Cross-
section decreased posterior shift of the center of pressure during gait propulsion. A decreased duration of the initial dorsiflexor muscle burst during gait initiation in both PD and the RBD groups.

Patients with RBD and PD FOG patients had

\section{Chahine et}

al. 2014 [44] 65 PD

Crosssection

Higher amounts of surface EMG activity were associated with longer PD disease duration and greater disease severity.

RBD patients have increased variability of trunk acceleration and decreased smoothness of sway,

Chen et al. 2014 [40]

Crosssection especially during challenging obstacles. RBD patients demonstrated significant impairment in stereopsis.

Tonic muscle activity was increased significantly in

Videnovic et al. 2013 [14]

Crossthe RBD and FOG groups. Phasic muscle activity section was significantly increased in the RBD group compared to all other groups.

42 RBD,

McDade et al. 2013 [39] controls

Cross-

Probable RBD was associated with decreased section velocity, cadence, significantly increased double limb support variability, greater stride time variability, and swing time variability.

Patients with PD and RBD have fewer tremor

Postuma et al. 2008 [38]
36 PD symptoms. An increased frequency of falls was section noted among patients with RBD and are less

responsive to their medications.

People with RBD show alterations in the coupling of posture and gait similar to those seen in PD even before diagnosing a degenerative disorder.

Surface EMG activity during REM sleep was associated with the severity of both PD and RBD. This EMG result may be useful as a PD biomarker.

Idiopathic RBD patients, particularly those with abnormal stereopsis, have subliminal postural instability under challenging conditions. Postural sway performance can be a biological marker for PD diagnosis.

These findings provide evidence that increased muscle activity during REM sleep is a co-morbid feature of patients with PD with FOG.

Probable RBD is associated with subtle gait changes before PD diagnosis. Diagnosis of probable RBD supplemented by gait analysis may help as a screening tool for the diagnosis of synucleinopathies.

The presence of altered motor subtypes in PD with RBD suggests a different neurodegeneration pattern compared to PD patients without RBD.

\section{TABLE 1: Summary of all the articles reviewed in this study}

PD - Parkinson's disease; STN - subthalamic nucleus; FOG - freezing of gait; RCT - randomized controlled trial; CLR - cerebellar locomotor region; DBS - deep brain stimulation; PPN - pedunculopontine nucleus; SMA - supplementary motor area; ACC - anterior cingulate cortex; REM - rapid eye movement; RBD - rapid eye movement sleep behavior disorder; MLR - motor locomotor region; RSWA - rapid eye movement sleep without atonia; EMG - electromyography; APA - anticipatory postural adjustments; iRBD - idiopathic rapid eye movement sleep behavior disorder

\section{Limitations}

This review consisted mostly of cross-sectional studies and a few randomized controlled trials. Longitudinal studies to establish the relationship between RBD and FOG are lacking. In addition, only articles written in English were included in this review; hence, failing to include other studies that may have been relevant to the study.

\section{Conclusions}

This review reveals that the functional neuroanatomy that controls REM sleep, arousal, and locomotion overlap significantly. There is no single neural structure localized that causes both RBD and FOG in PD. Instead, a decreased functionality was identified heterogeneously in these neural networks notably in the cortical-subthalamic network, connections between the PPN and cerebellar locomotor region, PPN and SMA, as well as in the PPN to MPFC and PNN to ACC connectivities. Sensory alterations, particularly visual perception dysfunction is evident in $\mathrm{PD}$ and $\mathrm{RBD}$, which predisposes to increased motor symptoms in these patients. These motor symptoms that primarily affect gait initiation are common to both patients with RBD and FOG in PD, which subsequently leads to episodes of freezing. These findings are from cross-sectional studies published to date. Prospective studies are needed to establish all these findings' temporality and better understand the pathophysiology common to RBD and PD FOG. This approach allows better treatment approaches in PD patients with FOG and coexisting RBD and develop early diagnostic tools to diagnose PD with the FOG subtype.

\section{Additional Information}

\section{Disclosures}


Conflicts of interest: In compliance with the ICMJE uniform disclosure form, all authors declare the following: Payment/services info: All authors have declared that no financial support was received from any organization for the submitted work. Financial relationships: All authors have declared that they have no financial relationships at present or within the previous three years with any organizations that might have an interest in the submitted work. Other relationships: All authors have declared that there are no other relationships or activities that could appear to have influenced the submitted work.

\section{References}

1. Boeve BF: REM sleep behavior disorder: Updated review of the core features, the REM sleep behavior disorder-neurodegenerative disease association, evolving concepts, controversies, and future directions. Ann N Y Acad Sci. 2010, 1184:17-56.

2. Schenck $\mathrm{CH}$, Boeve BF, Mahowald MW: Delayed emergence of a parkinsonian disorder or dementia in $81 \%$ of older men initially diagnosed with idiopathic rapid eye movement sleep behavior disorder: a 16-year update on a previously reported series. Sleep Med. 2013, 14:744-748. 10.1016/j.sleep.2012.10.009

3. Braak H, Del Tredici K, Rüb U, de Vos RA, Jansen Steur EN, Braak E: Staging of brain pathology related to sporadic Parkinson's disease. Neurobiol Aging. 2003, 24:197-211. 10.1016/s0197-4580(02)00065-9

4. Boeve BF, Silber MH, Saper CB, et al.: Pathophysiology of REM sleep behaviour disorder and relevance to neurodegenerative disease. Brain. 2007, 130:2770-2788. 10.1093/brain/awm056

5. Gagnon JF, Bédard MA, Fantini ML, et al.: REM sleep behavior disorder and REM sleep without atonia in Parkinson's disease. Neurology. 2002, 59:585-589. 10.1212/wnl.59.4.585

6. Sixel-Döring F, Trautmann E, Mollenhauer B, Trenkwalder C: Associated factors for REM sleep behavior disorder in Parkinson's disease. Neurology. 2011, 77:1048-1054. 10.1212/WNL.0b013e31822e560e

7. Postuma RB, Gagnon JF, Vendette M, Charland K, Montplaisir J: REM sleep behaviour disorder in Parkinson's disease is associated with specific motor features. J Neurol Neurosurg Psychiatry. 2008, 79:11171121. 10.1136/jnnp.2008.149195

8. Romenets SR, Gagnon JF, Latreille V, et al.: Rapid eye movement sleep behavior disorder and subtypes of Parkinson's disease. Mov Disord. 2012, 27:996-1003.

9. Iansek R, Danoudis M: Freezing of gait in Parkinson's disease: its pathophysiology and pragmatic approaches to management. Mov Disord Clin Pract. 2016, 4:290-297. 10.1002/mdc3.12463

10. Benarroch EE: Pedunculopontine nucleus: functional organization and clinical implications . Neurology. 2013, 80:1148-1155. 10.1212/WNL.0b013e3182886a76

11. Nutt JG, Bloem BR, Giladi N, Hallett M, Horak FB, Nieuwboer A: Freezing of gait: moving forward on a mysterious clinical phenomenon. Lancet Neurol. 2011, 10:734-744. 10.1016/S1474-4422(11)70143-0

12. Snijders AH, Leunissen I, Bakker M, et al.: Gait-related cerebral alterations in patients with Parkinson's disease with freezing of gait. Brain. 2011, 134:59-72. 10.1093/brain/awq324

13. Luppi PH, Clément O, Sapin E, et al.: The neuronal network responsible for paradoxical sleep and its dysfunctions causing narcolepsy and rapid eye movement (REM) behavior disorder. Sleep Med Rev. 2011, 15:153-163. 10.1016/j.smrv.2010.08.002

14. Videnovic A, Marlin C, Alibiglou L, Planetta PJ, Vaillancourt DE, Mackinnon CD: Increased REM sleep without atonia in Parkinson disease with freezing of gait. Neurology. 2013, 81:1030-1035. 10.1212/WNL.0b013e3182a4a408

15. MacKinnon CD, Alibiglou L, Videnovic A: Gait and postural disorders in REM sleep behavior disorder . Springer, Cham; 2019.

16. Steriade M, Datta S, Paré D, Oakson G, Curró Dossi RC: Neuronal activities in brainstem cholinergic nuclei related to tonic activation processes in thalamocortical systems. J Neurosci. 1990, 10:2541-2559. 10.1523/JNEUROSCI.10-08-02541.1990

17. Datta S, Siwek DF: Single cell activity patterns of pedunculopontine tegmentum neurons across the sleepwake cycle in the freely moving rats. J Neurosci Res. 2002, 70:611-621. 10.1002/jnr.10405

18. Garcia-Rill E, Homma Y, Skinner RD: Arousal mechanisms related to posture and locomotion: 1. Descending modulation. Prog Brain Res. 2004, 143:283-290. 10.1016/S0079-6123(03)43027-6

19. Takakusaki K, Obara K, Nozu T, Okumura T: Modulatory effects of the GABAergic basal ganglia neurons on the PPN and the muscle tone inhibitory system in cats. Arch Ital Biol. 2011, 149:385-405.

20. Weng FJ, Williams RH, Hawryluk JM, et al.: Carbachol excites sublaterodorsal nucleus neurons projecting to the spinal cord. J Physiol. 2014, 592:1601-1617. 10.1113/jphysiol.2013.261800

21. Sinnamon HM, Jassen AK, Vita LA: Brainstem regions with neuronal activity patterns correlated with priming of locomotor stepping in the anesthetized rat. Neuroscience. 2000, 99:77-91.

22. Takakusaki K, Chiba R, Nozu T, Okumura T: Brainstem control of locomotion and muscle tone with special reference to the role of the mesopontine tegmentum and medullary reticulospinal systems. J Neural Transm. 2016, 123:695-729. 10.1007/s00702-015-1475-4

23. Takakusaki K: Functional neuroanatomy for posture and gait control. J Mov Disord. 2017, 10:1-17. 10.14802/jmd.16062

24. Amboni M, Barone P, Iuppariello L, et al.: Gait patterns in Parkinsonian patients with or without mild cognitive impairment. Mov Disord. 2012, 27:1536-1543.

25. Jahn K, Deutschländer A, Stephan T, et al.: Imaging human supraspinal locomotor centers in brainstem and cerebellum. Neuroimage. 2008, 39:786-792. 10.1016/j.neuroimage.2007.09.047

26. Snijders AH, Takakusaki K, Debu B, et al.: Physiology of freezing of gait. Ann Neurol. 2016, 80:644-659. 10.1002/ana.24778

27. Pozzi NG, Canessa A, Palmisano C, et al.: Freezing of gait in Parkinson's disease reflects a sudden derangement of locomotor network dynamics. Brain. 2019, 142:2037-2050. 10.1093/brain/awz141

28. Fling BW, Cohen RG, Mancini M, Nutt JG, Fair DA, Horak FB: Asymmetric pedunculopontine network connectivity in parkinsonian patients with freezing of gait. Brain. 2013, 136:2405-2418. 10.1093/brain/awt172

29. Fasano A, Laganiere SE, Lam S, Fox MD: Lesions causing freezing of gait localize to a cerebellar functional 
network. Ann Neurol. 2017, 81:129-141. 10.1002/ana.24845

30. Wang M, Jiang S, Yuan Y, et al.: Alterations of functional and structural connectivity of freezing of gait in Parkinson's disease. J Neurol. 2016, 263:1583-1592. 10.1007/s00415-016-8174-4

31. Gallea C, Ewenczyk C, Degos B, et al.: Pedunculopontine network dysfunction in Parkinson's disease with postural control and sleep disorders. Mov Disord. 2017, 32:693-704. 10.1002/mds.26923

32. Fling BW, Cohen RG, Mancini M, et al.: Functional reorganization of the locomotor network in Parkinson patients with freezing of gait. PLoS One. 2014, 9:100291. 10.1371/journal.pone.0100291

33. Factor SA, Scullin MK, Sollinger AB, et al.: Freezing of gait subtypes have different cognitive correlates in Parkinson's disease. Parkinsonism Relat Disord. 2014, 20:1359-1364. 10.1016/j.parkreldis.2014.09.023

34. Almeida QJ, Lebold CA: Freezing of gait in Parkinson's disease: a perceptual cause for a motor impairment? . J Neurol Neurosurg Psychiatry. 2010, 81:513-518. 10.1136/jnnp.2008.160580

35. Marques A, Dujardin K, Boucart M, et al.: REM sleep behaviour disorder and visuoperceptive dysfunction: a disorder of the ventral visual stream?. J Neurol. 2010, 257:383-391. 10.1007/s00415-009-5328-7

36. Plomhause L, Dujardin K, Boucart M, et al.: Impaired visual perception in rapid eye movement sleep behavior disorder. Neuropsychology. 2014, 28:388-393. 10.1037/neu0000006

37. Fearon C, Butler JS, Newman L, Lynch T, Reilly RB: Audiovisual processing is abnormal in Parkinson's disease and correlates with freezing of gait and disease duration. J Parkinsons Dis. 2015, 5:925-936. 10.3233/JPD-150655

38. Postuma RB, Gagnon JF, Vendette M, Charland K, Montplaisir J: REM sleep behaviour disorder in Parkinson's disease is associated with specific motor features. J Neurol Neurosurg Psychiatry. 2008, 79:11171121. 10.1136/jnnp.2008.149195

39. McDade EM, Boot BP, Christianson TJ, et al.: Subtle gait changes in patients with REM sleep behavior disorder. Mov Disord. 2013, 28:1847-1853. 10.1002/mds.25653

40. Chen TZ, Xu GJ, Zhou GA, Wang JR, Chan P, Du YF: Postural sway in idiopathic rapid eye movement sleep behavior disorder: a potential marker of prodromal Parkinson's disease. Brain Res. 2014, 1559:26-32. 10.1016/j.brainres.2014.02.040

41. Alibiglou L, Videnovic A, Planetta PJ, Vaillancourt DE, MacKinnon CD: Subliminal gait initiation deficits in rapid eye movement sleep behavior disorder: A harbinger of freezing of gait?. Mov Disord. 2016, 31:17111719. $10.1002 / \mathrm{mds} .26665$

42. Massion J: Movement, posture and equilibrium: interaction and coordination. Prog Neurobiol. 1992, 38:3556. 10.1016/0301-0082(92)90034-c

43. Thevathasan W, Cole MH, Graepel CL, et al.: A spatiotemporal analysis of gait freezing and the impact of pedunculopontine nucleus stimulation. Brain. 2012, 135:1446-1454. 10.1093/brain/aws039

44. Chahine LM, Kauta SR, Daley JT, Cantor CR, Dahodwala N: Surface EMG activity during REM sleep in Parkinson's disease correlates with disease severity. Parkinsonism Relat Disord. 2014, 20:766-771. 10.1016/j.parkreldis.2014.04.011

45. Linn-Evans ME, Petrucci MN, Amundsen Huffmaster SL, et al.: REM sleep without atonia is associated with increased rigidity in patients with mild to moderate Parkinson's disease. Clin Neurophysiol. 2020, 131:20082016. 10.1016/j.clinph.2020.04.017 